\title{
SOMEWHAT SMOOTH NUMBERS IN SHORT INTERVALS
}

\author{
ANDREAS WEINGARTNER
}

\begin{abstract}
We use exponent pairs to establish the existence of many $x^{a}$-smooth numbers in short intervals $\left[x-x^{b}, x\right]$, when $a>1 / 2$. In particular, $b=1-a-a(1-a)^{3}$ is admissible. Assuming the exponentpairs conjecture, one can take $b=(1-a) / 2+\epsilon$. As an application, we show that $\left[x-x^{0.4872}, x\right]$ contains many practical numbers when $x$ is large.
\end{abstract}

\section{INTRODUCTION}

We say that a natural number $n$ is $y$-smooth if all of its prime factors are $\leq y$. Let $\Psi(x, y)$ be the number of such $n \leq x$. Improving on many earlier efforts by a number of different authors, Matomäki and Radziwiłł[10] established the existence of many $x^{\epsilon}$-smooth numbers in intervals of the form $[x, x+c(\epsilon) \sqrt{x}]$, for every $\epsilon>0$. Harman [6] showed that intervals around $x$ of length $x^{0.45 \cdots}$ contain many $x^{0.27 \cdots}$-smooth numbers.

We are interested in the existence of $x^{a}$-smooth numbers in much shorter intervals, when $a>1 / 2$. More precisely, given $a \in(1 / 2,1)$, how small can we take $b$ such that

$$
\Psi\left(x, x^{a}\right)-\Psi\left(x-x^{b}, x^{a}\right) \gg x^{b-\epsilon}
$$

for every $\epsilon>0$ ? In that direction, Friedlander and Lagarias [3] showed that there exists a constant $c>0$ such that $b=1-a-c a(1-a)^{3}$ is admissible, even with $\epsilon=0$, but without providing any numerical estimate for $c$. We will use exponent pairs (see [4]) to find explicit values of $b<1-a$. In particular, $b=1-a-a(1-a)^{3}$ is admissible for every $a \in(1 / 2,1)$.

Let $\psi(x)=x-\lfloor x\rfloor-1 / 2=\{x\}-1 / 2$. The method used by Friedlander and Lagarias [3] starts with Chebyshev's identity and requires estimates for sums of $\psi(x / p) \log p$, where $p$ runs over primes. Our approach involves sums of $\psi(x / n)$ over all integers $n$ from an interval. We use the estimate

$$
\sum_{N \leq n \leq 2 N} \psi(x / n) \ll \min \left(x^{\theta}, x^{k /(k+1)} N^{(l-k) /(k+1)}\right) \quad(1 \leq N \leq \sqrt{x}),
$$

where $(k, l)$ is any exponent pair. The two most recent records for $\theta$ are $\theta=\frac{131}{416}+\epsilon=0.3149 \ldots$ by Huxley $\left[9\right.$, Thm. 4] and $\theta=\frac{517}{1648}+\epsilon=0.3137 \ldots$ by Bourgain and Watt [2, Eq. (7.4)]. For the second estimate in (1), see Graham and Kolesnik [4, Lemma 4.3].

Date: August 18, 2021.

2010 Mathematics Subject Classification. 11N25. 
Let $\nu=2.9882 \ldots$ be the minimum value of $\left(2^{u}-1\right) /(u-1)$ for $u>1$.

Theorem 1. Let $(k, l)$ be an exponent pair and $\theta$ as in (1). There is a constant $K$ such that

$$
\Psi(x, y)-\Psi(x-z, y) \gg \frac{z}{(\log x)^{\nu}},
$$

provided $x \geq y \geq \sqrt{2 x}$ and $x \geq z \geq K \min \left(x^{\theta}, x^{l /(k+1)} y^{(k-l) /(k+1)}\right)$.

Define

$$
b=b(a, k, l)=\frac{l+a(k-l)}{k+1} .
$$

Corollary 1. Let $(k, l)$ be an exponent pair, $\theta$ as in (1) and $1 / 2<a \leq 1$. There is a constant $K$ such that for $x \geq z \geq K x^{\min (\theta, b)}$,

$$
\Psi\left(x, x^{a}\right)-\Psi\left(x-z, x^{a}\right) \gg \frac{z}{(\log x)^{\nu}} .
$$

If $a=1 / 2$, the conclusion holds if $x^{a}$ is replaced by $\sqrt{2 x}$.

Starting with the exponent pair $(\kappa, \lambda)=(13 / 84+\epsilon, 55 / 84+\epsilon)$ of Bourgain [1, Thm. 6], and possibly applying van der Corput's processes $A$ or $B$, we find a sequence of linear functions in $a$, shown in Table 1 . When $a$ is close to $1 / 2$, then $\theta$ is smaller than any $b$ obtained from known exponent pairs. When $a$ is close to 1 , we rely on exponent pairs $(k, l)$ with small $k$. Heath-Brown [8, Thm. 2] found that for integers $m \geq 3$ and every $\epsilon>0$,

$$
k_{m}=\frac{2}{(m-1)^{2}(m+2)}, \quad l_{m}=1-\frac{3 m-2}{m(m-1)(m+2)}+\epsilon
$$

is an exponent pair. This enables us to prove the following result.

Corollary 2. For each $a \in[1 / 2,1)$, the conclusion of Corollary 1 holds for some $b<1-a-a(1-a)^{3}-4.32 a(1-a)^{5}$.

The value of $a$, for which $b\left(a, k_{m}, l_{m}\right)=b\left(a, k_{m+1}, l_{m+1}\right)$, is given by

$$
a_{m}:=1-\frac{1}{m}+\frac{2-m^{-1}}{m^{3}+m^{2}+2 m-1}
$$

If $a>0.796 \ldots$ and $a \in\left[a_{m-1}, a_{m}\right]$, then $b$ is minimized by $b\left(a, k_{m}, l_{m}\right)$. This yields slightly smaller values of $b$ than Corollary 2 .

The values $a=1-1 / m$, where $m \geq 2$ is an integer, may be of particular interest. Here we have $a_{m-1}<a=1-1 / m<a_{m}$ and

$$
b=b\left(1-1 / m, k_{m}, l_{m}\right)=\frac{(m-1)\left(m^{3}+m^{2}-3 m+2\right)}{m^{2}\left(m^{3}-3 m+4\right)}+\epsilon .
$$

The exponent-pairs conjecture states that $(k, l)=(\epsilon, 1 / 2+\epsilon)$ is an exponent pair for every $\epsilon>0$.

Corollary 3. If $(\epsilon, 1 / 2+\epsilon)$ is an exponent pair, then the conclusion of Corollary 1 holds with $b=(1-a) / 2+\epsilon$ for each $a \in[1 / 2,1]$. 


\begin{tabular}{|c|c|c|}
\hline$b$ & Interval for $a$ & Exponent Pair \\
\hline $517 / 1648+\epsilon$ & {$[0.500 \ldots, 0.579 \ldots]$} & \\
\hline$(110-55 a) / 249+\epsilon$ & {$[0.579 \ldots, 0.590 \ldots]$} & $B A(\kappa, \lambda)$ \\
\hline$(55-42 a) / 97+\epsilon$ & {$[0.590 \ldots, 0.701 \ldots]$} & $(\kappa, \lambda)$ \\
\hline$(152-139 a) / 207+\epsilon$ & {$[0.701 \ldots, 0.766 \ldots]$} & $A(\kappa, \lambda)$ \\
\hline$(359-346 a) / 427+\epsilon$ & {$[0.766 \ldots, 0.796 \ldots]$} & $A A(\kappa, \lambda)$ \\
\hline$b\left(a, k_{m}, l_{m}\right)$ & {$\left[a_{m-1}, a_{m}\right], m \geq 5$} & $\left(k_{m}, l_{m}\right)$ \\
\hline
\end{tabular}

TABLE 1. Admissible values of $b$, depending on $a$.

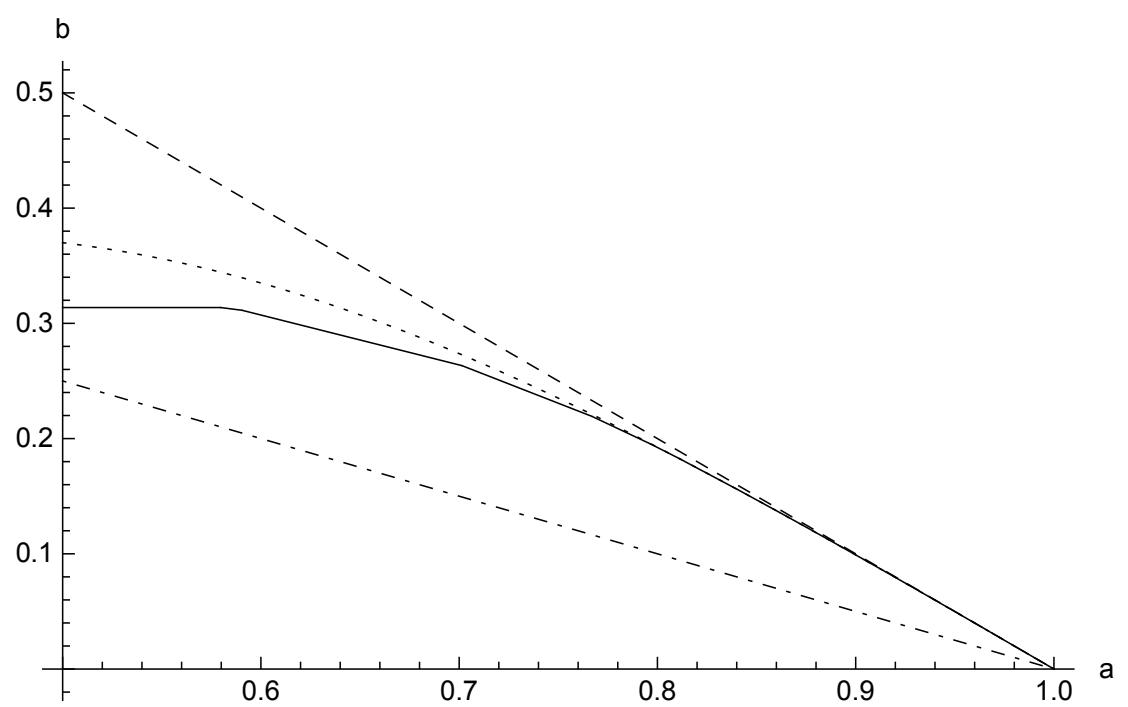

Figure 1. Admissible values of $b$ based on Table 1 (solid); $b=1-a-a(1-a)^{3}-4.32 a(1-a)^{5}$ (dotted) from Cor. 2; $b=1-a$ (dashed) from the exponent pair $(k, l)=(0,1)$; $b=\frac{1}{2}(1-a)$ (dot-dashed) from the exponent-pairs conjecture.

If one is only concerned with the existence of a single $y$-smooth number in short intervals, then a construction due to Friedlander and Lagarias [3] (consider integers of the form $m^{2}-h^{2}=(m-h)(m+h)$, where $m=\lceil\sqrt{x}\rceil$ and $h=0,1,2, \ldots$ ) and an easy exercise (aided by a computer to deal with small values of $x$ ) lead to the explicit estimate

$$
\Psi(x, \sqrt{2 x})-\Psi\left(x-3 x^{1 / 4}, \sqrt{2 x}\right) \geq 1 \quad(x \geq 1) .
$$

From Table 1, we find that our intervals are wider than $3 x^{1 / 4}$ when $a<$ $401 / 556=0.721 \ldots$, but are shorter when $a>401 / 556$. 


\section{Proofs}

Let $\tau(n)$ be the number of positive divisors of $n$. The following estimate is a special case of Theorem 2 of Shiu [16].

Lemma 1. Let $\epsilon>0$ and $u \in \mathbb{R}$ be fixed. For $x \geq 2$ and $x^{\epsilon} \leq z \leq x$, we have

$$
\sum_{x-z \leq n \leq x}(\tau(n))^{u} \ll z(\log x)^{2^{u}-1} .
$$

Proof of Theorem 1. Let $P(n)$ denote the largest prime factor of $n$. Note that the result holds if $z>x / 2$, so we may assume $z \leq x / 2$. Define

$$
S:=\sum_{x / y \leq d \leq 2 x / y} \sum_{\substack{x-z<n \leq x \\ n \equiv 0 \bmod d}} 1 .
$$

We have

$$
\begin{aligned}
S & =\sum_{x / y \leq d \leq 2 x / y}(\lfloor x / d\rfloor-\lfloor(x-z) / d\rfloor) \\
& =\sum_{x / y \leq d \leq 2 x / y} z / d-\sum_{x / y \leq d \leq 2 x / y} \psi(x / d)+\sum_{x / y \leq d \leq 2 x / y} \psi((x-z) / d) \\
& \geq z / 3+O\left(\min \left(x^{\theta}, x^{l /(k+1)} y^{(k-l) /(k+1)}\right)\right) \\
& \geq z / 4,
\end{aligned}
$$

by (1) and the assumptions of Theorem 1 .

Note that $y \geq \sqrt{2 x}$ implies $2 x / y \leq y$. Every $n$ counted in the inner sum of (4) has a divisor $d \in[x / y, 2 x / y] \subseteq[x / y, y]$. Since $d \leq y$ and $n / d \leq x /(x / y)=y$, we have $P(n) \leq y$, i.e. $n$ is $y$-smooth. Moreover, each $n$ is counted at most $\tau(n)$ times, once for each divisor $d$ of $n$ with $d \in[x / y, 2 x / y]$. Thus,

$$
S \leq \sum_{\substack{x-z<n \leq x \\ P(n) \leq y}} \tau(n) .
$$

For real numbers $t, u>1$ with $1 / t+1 / u=1$, Hölder's inequality yields

$$
\begin{aligned}
S & \leq\left(\sum_{\substack{x-z<n \leq x \\
P(n) \leq y}} 1\right)^{1 / t}\left(\sum_{x-z<n \leq x} \tau(n)^{u}\right)^{1 / u} \\
& \ll(\Psi(x, y)-\Psi(x-z, y))^{1 / t} z^{1 / u}(\log x)^{\left(2^{u}-1\right) / u},
\end{aligned}
$$

by Lemma 1 . Since $S \geq z / 4$, we get

$$
\Psi(x, y)-\Psi(x-z, y) \gg \frac{z}{(\log x)^{\left(2^{u}-1\right) /(u-1)}} .
$$

The last exponent has a minimum value of $\nu=2.9882 \ldots$ at $u=2.1080 \ldots$ 
Proof of Corollary 2. For $m \geq 3$ and $a \in\left[a_{m-1}, a_{m}\right]$, we want to show that $b\left(a, k_{m}, l_{m}\right)<f(a)$, where $f(a)=1-a-a(1-a)^{3}-4.32 a(1-a)^{5}$. Since $f^{\prime \prime}(a)<0$ for $1 / 2<a<1$ and $b\left(a, k_{m}, l_{m}\right)$ is a linear function in $a$ for each $m$, it suffices to verify the inequality at the endpoints $a=a_{m}$. That is, we need to show that $b\left(a_{m}, k_{m}, l_{m}\right)<f\left(a_{m}\right)$ for $m \geq 2$. We find that $f\left(a_{m}\right)-b\left(a_{m}, k_{m}, l_{m}\right)$ is a rational function in $m$ that is positive for every $m \geq 1$. This proves the claim for $a \geq a_{2}=3 / 5$. If $1 / 2 \leq a<3 / 5$, the result follows from Table 1.

\section{Application to practical numbers}

Let $\mathcal{A}$ be the set of positive integers containing $n=1$ and all those $n \geq 2$ with prime factorization $n=p_{1}^{\alpha_{1}} \cdots p_{k}^{\alpha_{k}}, p_{1}<p_{2}<\ldots<p_{k}$, which satisfy $p_{1}=2$ and

$$
p_{i} \leq p_{1}^{\alpha_{1}} \cdots p_{i-1}^{\alpha_{i-1}} \quad(2 \leq i \leq k)
$$

The significance of the set $\mathcal{A}$ is that it is a subset of several notable integer sequences, including the practical numbers (i.e. integers $n$ such that every natural number $m \leq n$ can be expressed as a sum of distinct positive divisors of $n[14,15,17,18]$ ), the $t$-dense numbers, for every $t \geq 2$, (i.e. the ratios of consecutive divisors of $n$ are at most $t,[14,15,17,18])$, and the $\varphi$-practical numbers (i.e. $x^{n}-1$ has a divisor in $\mathbb{Z}[x]$ of every degree up to $n,[12]$ ).

Let $\nu=2.9882 \ldots$ be as in Theorem $1, C=\left(1-e^{-\gamma}\right)^{-1}=2.280 \ldots$, where $\gamma=0.5772 \ldots$ is Euler's constant, and

$$
\mu_{0}:=2 \nu+2+C \log 2=9.5569 \ldots
$$

Theorem 2. Let $(k, l)$ be an exponent pair, $\beta=\frac{5 k+l+2}{6(k+1)}$ and $\mu>\mu_{0}$.

There exists a constant $K$ such that for $x \geq z \geq K x^{\beta}$, the interval $[x-z, x]$ contains $\gg z(\log x)^{-\mu}$ members of $\mathcal{A}$.

The exponent pair $(k, l)=(13 / 194+\epsilon, 76 / 97+\epsilon)=A(\kappa, \lambda)$ yields:

Corollary 4. For every $\beta>605 / 1242=0.4871 \ldots$ and $\mu>\mu_{0}$, the conclusion of Theorem 2 holds. Assuming the exponent-pairs conjecture, it holds for every $\beta>5 / 12=0.4166 \ldots$.

Corollary 5. The interval $\left[x-x^{0.4872}, x\right]$ contains at least $x^{0.4872}(\log x)^{-9.557}$ members of $\mathcal{A}$, for all sufficiently large $x$.

A quick search on a computer suggests that Corollary 5 probably holds for all $x \geq 504$.

It is clear that Theorem 2 and its corollaries remain valid if $\mathcal{A}$ is replaced by any superset of $\mathcal{A}$. In the case of practical numbers, Corollary 5 improves on two earlier results: Hausman and Shapiro [7] found that the interval $\left[x^{2},(x+1)^{2}\right]$ contains a practical number for every $x \geq 1$, in analogy with Legendre's conjecture for primes. Melfi [11, Thm. 9] sharpened this by showing that the interval $[x, x+K \sqrt{x / \log \log x}]$ contains a practical number for all large $x$ and some constant $K$. 
Granville [5, Conj. 4.4.2] states the conjecture that for every fixed $\epsilon>$ 0 , the interval $\left[x-x^{\epsilon}, x\right]$ contains a $x^{\epsilon}$-smooth number for all $x \geq x_{0}(\epsilon)$. Pomerance [13] points out that this would imply the existence of a practical number (or member of $\mathcal{A}$ ) in every interval $\left[x-x^{\epsilon}, x\right]$ for large $x$.

The following observation follows at once from the definition of the set $\mathcal{A}$.

Lemma 2. If $n \in \mathcal{A}$ and $P(m) \leq n$, then $m n \in \mathcal{A}$.

Proof of Theorem 2. If $z>x / 2$, the result follows from Theorem 1.2 of [18], so we may assume $z \leq x / 2$. Let $a=3 / 4$. We have $b=\frac{3 k+l}{4(k+1)}>0$, according to $(2)$, and $\beta=1 / 3+(2 / 3) b>1 / 3$.

Theorem 1.2 of [18] shows that the number of $n \in \mathcal{A} \cap\left(2 x^{1 / 3}, 3 x^{1 / 3}\right]$ is $\sim c x^{1 / 3} / \log x$ for some positive constant $c$. Let $\epsilon>0$ and $C=(1-$ $\left.e^{-\gamma}\right)^{-1}=2.280 \ldots$ By Corollary 1 of [19], the number of these $n$ with $\Omega(n)>(C+\epsilon) \log \log n$ is $o\left(x^{1 / 3} / \log x\right)$, so we may exclude such $n$ and assume $\Omega(n) \leq(C+\epsilon) \log \log n$.

Since $n \in\left(2 x^{1 / 3}, 3 x^{1 / 3}\right]$, the condition $z \geq 3 K x^{\beta}$ implies $z / n \geq K(x / n)^{b}$. By Corollary 1 , for each of these $n$, the interval $I_{n}:=[x / n-z / n, x / n]$ contains $\gg(z / n)(\log x / n)^{-\nu} \gg z x^{-1 / 3}(\log x)^{-\nu}$ integers $m$ that are $(x / n)^{3 / 4}$ smooth. Note that $m n \in[x-z, x]$ for $m \in I_{n}$.

We will show that for each of these pairs $(n, m)$ as described above, we have $m n \in \mathcal{A}$. Let $p=P(m)$. Since $n \geq 2 x^{1 / 3}, p \leq(x / n)^{3 / 4} \leq x^{1 / 2} 2^{-3 / 4}$. If $p \leq x^{1 / 3}$, then $m n \in \mathcal{A}$, by Lemma 2. If $p>x^{1 / 3}$, write $m=p r$ and note that $r=m / p \leq x /(n p)<x^{1 / 3}$. Thus, $r n \in \mathcal{A}$ by Lemma 2. Since $p \leq x^{1 / 2} 2^{-3 / 4}$, we have $p^{2} \leq x 2^{-3 / 2}<m n=p r n$ and hence $p<r n$. Thus, $m n=p r n \in \mathcal{A}$ also holds in this case, by Lemma 2 .

The number of pairs $(m, n)$ is $\gg z(\log x)^{-1-\nu}$, but several pairs may lead to the same product $m n$. We have $\tau(n) \leq 2^{\Omega(n)} \leq(\log x)^{C \log 2+\epsilon}$. By Lemma 1, we have $\sum_{m \in I_{n}} \tau(m) \ll(z / n) \log x$. Since the number of $m \in I_{n}$ that are $(x / n)^{3 / 4}$-smooth is $\gg(z / n)(\log x)^{-\nu}$, we have $\tau(m) \ll(\log x)^{\nu+1}$ for a positive proportion of them. Thus, we may assume $\tau(m) \ll(\log x)^{\nu+1}$, and therefore $\tau(m n) \ll(\log x)^{\nu+1+C \log 2+\epsilon}$. It follows that the number of distinct products $m n$ is

$$
\gg \frac{z(\log x)^{-1-\nu}}{(\log x)^{\nu+1+C \log 2+\epsilon}}=\frac{z}{(\log x)^{\mu_{0}+\epsilon}} .
$$

\section{REFERENCES}

[1] J. Bourgain, Decoupling, exponential sums and the Riemann zeta function, J. Amer. Math. Soc. 30 (2017), no. 1, 205-224.

[2] J. Bourgain and N. Watt, Mean square of zeta function, circle problem and divisor problem revisited, Preprint, (2017), arXiv:1709.04340.

[3] J. B. Friedlander and J. C. Lagarias, On the distribution in short intervals of integers having no large prime factor, J. Number Theory 25 (1987), no. 3, 249-273. 
[4] S. W. Graham and G. Kolesnik, Van der Corput's method of exponential sums, London Mathematical Society Lecture Note Series, 126. Cambridge University Press, Cambridge, 1991.

[5] A. Granville, Smooth numbers: computational number theory and beyond, https: //dms.umontreal.ca/ andrew/PDF/msrire.pdf

[6] G. Harman, Integers without large prime factors in short intervals and arithmetic progressions, Acta Arith. 91 (1999), no. 3, 279-289.

[7] M. Hausman and H. N. Shapiro, On practical numbers, Comm. Pure Appl. Math. 37 (1984), no. 5, 705-713.

[8] D. R. Heath-Brown, A new kth derivative estimate for a trigonometric sum via Vinogradov's integral, English version published in Proc. Steklov Inst. Math. 296 (2017), no. 1, 88-103. Tr. Mat. Inst. Steklova 296 (2017), Analiticheskaya i Kombinatornaya Teoriya Chisel, 95-110.

[9] M. N. Huxley, Exponential sums and lattice points. III, Proc. London Math. Soc. (3) 87 (2003), no. 3, 591-609.

[10] K. Matomäki and M. Radziwiłł, Multiplicative functions in short intervals, Ann. of Math. (2) 183 (2016), no. 3, 1015-1056.

[11] G. Melfi, A survey on practical numbers, Rend. Sem. Mat. Univ. Politec. Torino 53 (1995), no. 4, 347-359.

[12] C. Pomerance, L. Thompson and A. Weingartner, On integers $n$ for which $X^{n}-1$ has a divisor of every degree. Acta Arith. 175 (2016), no. 3, 225-243.

[13] C. Pomerance, Practical numbers, Number Theory Web Seminar (via Zoom), August 13, 2020. https://math.dartmouth.edu/ carlp/practicaltalk.pdf

[14] C. Pomerance and A. Weingartner, On primes and practical numbers, Ramanujan J. (2021). https://doi.org/10.1007/s11139-020-00354-y

[15] E. Saias, Entiers à diviseurs denses 1, J. Number Theory 62 (1997), 163-191.

[16] P. Shiu, A Brun-Titchmarsh theorem for multiplicative functions, J. Reine Angew. Math. 313 (1980), 161-170.

[17] G. Tenenbaum, Sur un problème de crible et ses applications, Ann. Sci. École Norm. Sup. (4) 19 (1986), 1-30.

[18] A. Weingartner, Practical numbers and the distribution of divisors, Q. J. Math. 66 (2015), no. 2, 743-758.

[19] A. Weingartner, The number of prime factors of integers with dense divisors, preprint arXiv:2101.11585.

Department of Mathematics, 351 West University Boulevard, Southern Utah University, Cedar City, Utah 84720, USA

Email address: weingartner@suu.edu 